\title{
Papilledema in Overweight Patients
}

\author{
Essam E. Saber, Ayser A. Fayed, Marwa A. Tabl, Ingy M. El Barki
}

Department of Ophthalmology, Faculty of Medicine Benha University, Egypt.

Correspondence to: Ingy M. El Barki, Department of Ophthalmology, Faculty of Medicine Benha University, Egypt.

Email:

ingymohamed2019@gmail.com

Received: 28 November 2019

Accepted: 21 October 2021

\begin{abstract}
While there has been great progress in our understanding of obesity and Idiopathic Intracranial Hypertension (IIH) nearly 80 years ago, there is still much to be learned about the etiology, pathophysiology, and exact role of obesity in IIH, particularly within the pediatric population. The continued modification of the diagnostic criteria for IIH reflects this progress and will likely help physicians make more a more accurate diagnosis. We continue to see a strong association between IIH and recent weight gain, but encouragingly the converse appears to be true, as a similar amount of weight loss results in significant improvement. For the morbidly obese IIH patient, bariatric surgery is a likely effective treatment when noninvasive strategies have failed. Currently, studies of obesity markers in IIH patients have been inconclusive. More studies are needed for more
\end{abstract} association between obesity \& IIH \& role of obesity management to reduce IIH

Key words: Papilledema; Overweight; Obesity; IIH

\section{Introduction}

Papilledema is one of the most alarming signs in clinical medicine. Papilledema specifically refers to swelling of the optic disc resulting from increased intracranial pressure (ICP). The essential histological features of papilledema are optic disc edema, neuronal swelling, venous and capillary dilatation, an abnormal protrusion of the optic nerve head toward the vitreous, lateral displacement of the adjacent retina, and folds of the posterior retinal layers. Various hypotheses have been proposed over the years, including direct infiltration of the optic nerve head by CSF, obstruction of posterior flow of intraocular fluid, inherent turgescence of prelaminar nerve substance, swelling of glia, absence of the restraining influence of Muller cells in the peri-papillary retina, disequilibrium of hydrostatic pressure in the tissue and bloodstream, and compression of the central retinal vein as 
it traverses the subarachnoid space or cavernous sinus with elevated central venous pressure (1).

Research to date suggests that there are three components required for the development of papilledema: increased and fluctuating pressure in the distal optic nerve sheath; elevated central retinal venous pressure; and impaired perfusion of the nerve fibers traversing the lamina cribrosa. Despite the findings described above, numerous questions still exist regarding the pathogenesis of papilledema. The definition of Idiopathic Intracranial Hypertension (IIH) has evolved with clinical experience and advances in imaging technology. IIH can be diagnosed only if the following criteria are met: 1) symptoms and signs attributable to increased ICP or papilledema; 2) elevated ICP recorded during lumbar puncture in the lateral decubitus position; 3) normal CSF composition; 4) no imaging evidence of ventriculomegaly or a structural cause for increased ICP, such as a brain parenchymal, ventricular, meningeal, or venous sinus abnormality; and 5) no other cause of intracranial hypertension identified, such as use of certain medications. Several clinical series have identified factors that may influence visual outcome in patients with IIH.
Recent weight gain is one of the most important of these factors. (2)

The pathophysiology of IIH in obese people is linked to an increase in intracranial venous pressure and impaired venous return due to obstructive sleep apnea (forced inspiration against a closed glottis). In addition, obstructive sleep apnea leads to retention of carbon dioxide. This hypercapnia can induce cerebral venous dilation and transient elevations in intracranial pressure. IIH patients with overweight should be closely monitored for progression of visual field loss. If progression is seen, early consideration for definitive surgical intervention may be especially important in IIH patients with multiple risk factors for severe vision loss, including black race, male sex, anemia, hypertension, and very high BMI. While it has been definitively shown that aggressive efforts at weight loss often lead to remission of the disease, whether these efforts also reduce the risk of severe visual loss in very obese IIH patients remains to be determined. (3)

\section{Aim of the work \\ Aim of the work is to review the correlation between body over weight and progression of optic disc oedema.}




\section{Methods}

This is a review article, The search was performed in MEDLINE, Embase, Pubmed and CINAHL Plus in the same date range with the following medical terms: "Papilledema; Overweight; Obesity; IIH", including articles from 2000 to 2019, Excluded articles from review are those of language other than English.

\section{Results}

\section{Pathogenesis of papilloedema}

Papilloedema is swelling of the optic nerve head secondary to increased intracranial pressure. It is nearly always bilateral, although it may be asymmetrical. All other causes of disc edema not associated with raised intracranial pressure referred as "disc swelling" and usually produce visual impairment (4).

An impairment of the return of venous blood flow from the optic nerve as result of increased intracranial pressure plays a major role, as well as interruption of the axoplasmic flow, which normally occurs from the retina into the disc and optic nerve, may cause axonal swelling (5).

Not all patients with raised intracranial pressure will necessary develop papilloedema. Patients with a history of papilloedema before may develop substantial increase intracranial pressure but without obvious disc swelling because of glial scarring of the optic nerve head (4).

\section{Stages of papilledema}

1.Early papilloedema:

- It may be difficult to diagnose with certainty.

- Its main features:

- Visual symptoms are absent and visual acuity is normal.

- Optic disc shows hyperaemia and mild elevation.

- Indistinct disc margins and swelling of peri-papillary nerve fiber layer. The nasal margin is involved first, followed by the superior, inferior, and temporal (figure 1).

- There is loss of pervious spontaneous venous pulsation.

- However, as about $20 \%$ of normal don't show spontaneous venous pulsation, its absence doesn't necessarily mean that the intracranial pressure is raised. If venous pulsation is well preserved the diagnosis of papilloedema is unlikely (4). 


\section{Established papilloedema:}

- Transient visual loss obstruction in one or both eyes, lasting a few, often on standing may be present.

- Visual acuity normal or reduced.

- Optic disc shows sever hyperemia, moderate elevation with indistinct margins, which may be initially asymmetrical.

- $\quad$ The optic cup \& the small vessels traversing the disc are obscured.

- There is venous engorgement, parapapillary flame shaped Hemorrhages (Figure 2) and frequently also cottonwool spots.

- Hard exudates may radiate from the center of fovea in the form of a "macular fan" an incomplete star with the temporal part missing.

- $\quad$ The blind spot is enlarged.

\section{Chronic papilloedema (Figure 3):}

- Visual acuity is variable and the visual field being to be constricted.

- Optic discs are markedly elevated with a "champagne cork" appearance

- Cotton wool spots and hemorrhages are absent.
- Optociliary shunt and drusen like crystalline deposits may be present on the disc surface.

\section{Atrophic (secondary optic atrophy):}

In this stage visual acuity is severely impaired. Optic discs are white, slightly elevated, crossing blood vessels are few and the margin are indistinct (figure 4).

This appearance is also referred to as secondary optic atrophy and is most frequently seen in patient with history of treated cerebral tumors or benign intracranial hypertension (4).

The lamina cribrosa separates the optic nerve into its intraocular and retroocular portions. This anatomic subdivision is of physiologic significance, because under normal circumstances the tissue pressure within the intra ocular portion of the optic nerve is much higher than the posterior lamina. This normal pressure gradient at level of the lamina reflects the influence of the intraocular pressure, which exceeds the cerebrospinal fluid pressure.

Edema of the disc may occur when prelaminar tension is abruptly elevated in acute glaucoma; therefore other factors must be considered. The majority of cases of passive swelling of optic disc may be, therefore, classified as: 
(1) Pre-laminar (usually unilateral papilloedema), caused by intraocular disease.

(2) Retrolaminar (usually bilateral papilloedema), caused by intraorbital, intracranial, or spinal cord disease (6).

Idiopathic intracranial hypertension is a syndrome in which there is increased intracranial pressure (ICP) of unknown etiology. Previously known as "pseudotumor cerebri” or "benign intracranial hypertension", this condition most frequently occurs in obese women of childbearing age. IIH is more common in women and obese individuals. Obese women with IIH might have a preferential accumulation of fat in the lower body relative to other obese women in the same age range.

Overweight and obesity are defined as "abnormal or excessive fat accumulation that presents a risk to health". Overweight and obesity are major risk factors for a number of chronic diseases, including; diabetes, cardiovascular diseases and cancer. Obesity is a leading preventable cause of death worldwide and its prevalence is increasing at an alarming rate. Obesity is most common in children and adult, women, minorities, rural populations and low income groups, but is increasing in all socioeconomic groups.
As the prevalence of obesity has increased in the United States, defining the association between obesity and IIH has become increasingly more urgent. In the most recent surveys among African countries, Egypt has the highest prevalence of overweight (44\%) and obesity (39\%). While obesity has not been shown to be a cause of IIH, obesity is clearly associated with IIH. The typical patient with IIH has been described as a young obese female, as the incidence of $\mathrm{IIH}$ rises to 20 per 100,000 in obese females. Interestingly, the risk of IIH increases with increasing BMI and morbidly obese patients with IIH may have even worse visual outcomes. Thus, morbidly obese individuals may be at particularly high risk for severe and permanent visual loss from IIH.

The pathophysiology of obesity and increased intracranial pressure is undetermined and the cause of IIH is unknown

Several reports support the notion that recent weight gain contributes to the development of IIH found that weight gain in previously non obese patients was as much of a risk factor for development of IIH as obesity itself.

The fact that weight loss improves or resolves signs and symptoms of IIH 
supports the strong association of obesity and IIH.

While the etiology of IIH remains unknown, there have been many postulated theories. Proposed mechanisms that could explain the elevated ICP in IIH include increased cerebral volume (either interstitial fluid, blood or tissue), increased CSF volume due to increased production or resistance to CSF outflow, loss of cerebral autoregulation resulting in elevated cerebral arterial pressure or increased cerebral venous pressure leading to increased CSF volume and decreased CSF outflow.

There is still uncertainty as to whether the degree of obesity affects the severity of symptoms and disease outcomes, particular visual field defects. According to multiple studies, it appears that those with morbid obesity (BMI >40) have worse visual outcomes and this further underscores the importance of aggressively pursuing weight loss options in extremely obese patients.
As a whole, the data supports the role of weight loss in the treatment of IIH. Although patients may require other treatment modalities, given the benefits of even modest weight loss on IIH disease progression and outcomes, this should be an area of emphasis for treating physicians.

Weight loss can lead to remission of the symptoms of IIH. Management includes, but is not limited to, dietary and behavior modification incorporating routine physical activity. In fact, PTC/IIH in the setting of obesity may be curative with body weight loss of $10 \%$.

Although a number of treatments have been advocated, currently there is no evidence-based management strategy. Symptom relief is the primary goal of management, but achieving this goal and preventing morbidity may require a multifocal approach. Preserving visual function and headache relief- are priorities. Best practice would include an interdisciplinary team collaborating closely with a neurologist and ophthalmologist. 


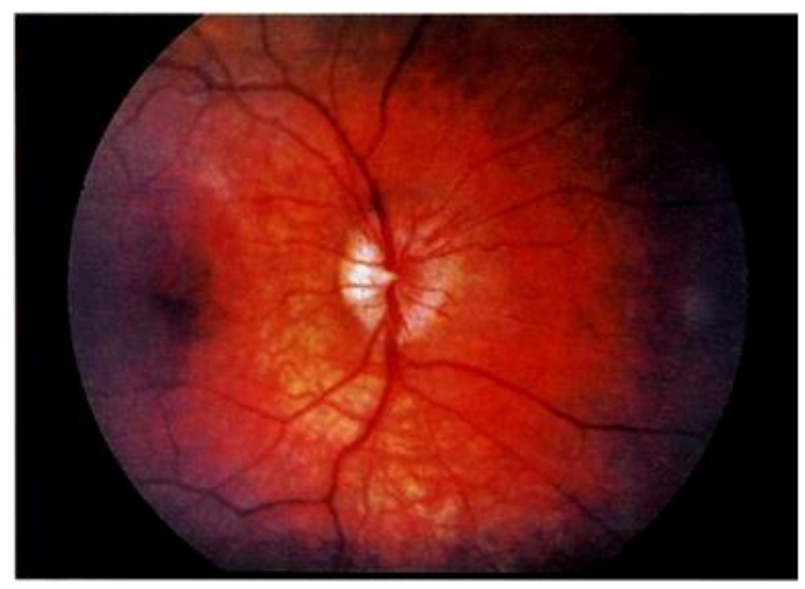

Figure (1): Early Papilloedema (4)

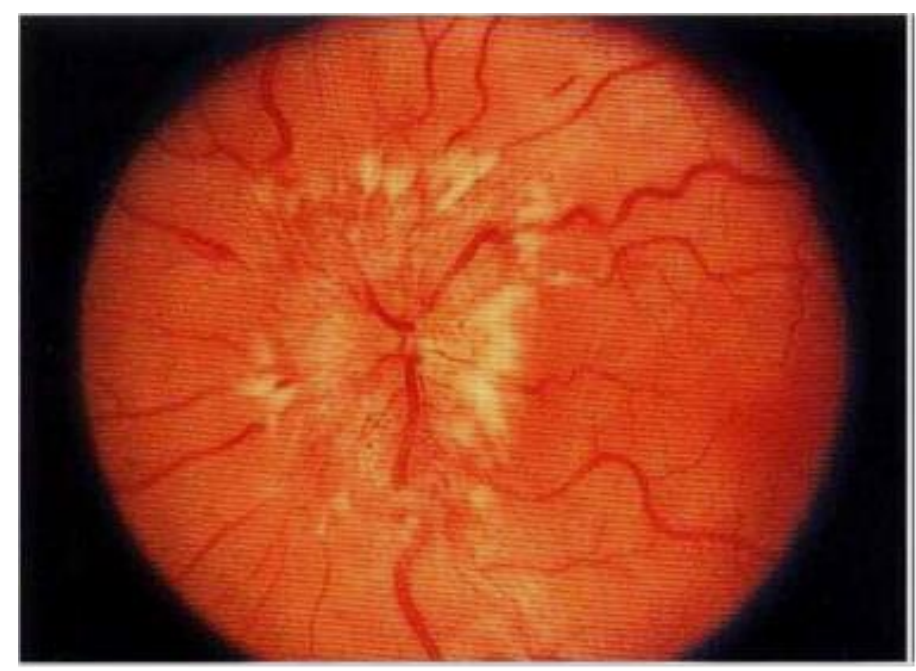

Figure (2): Established papilloedema (4).

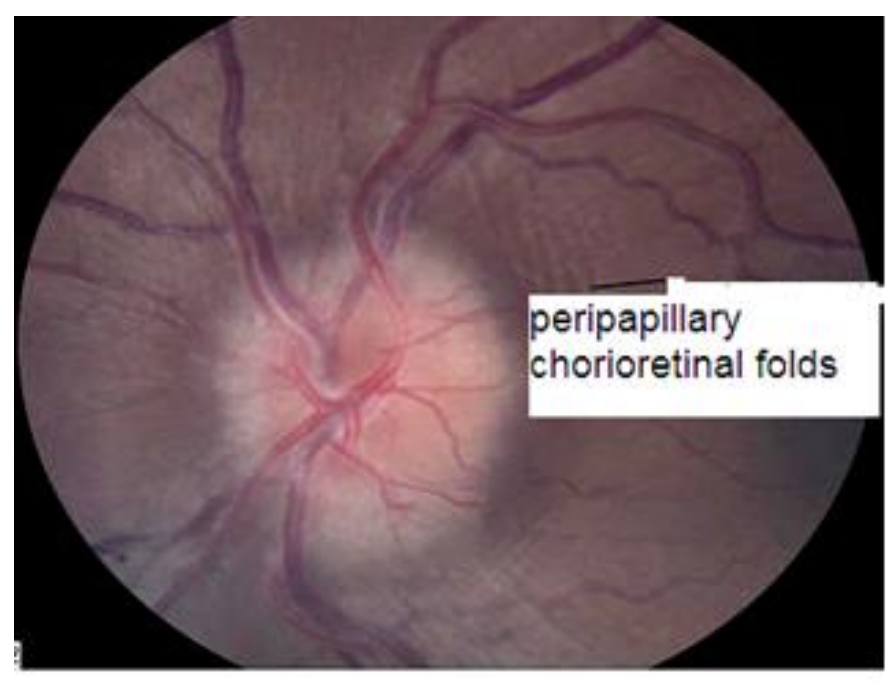

Figure (3): Chronic papilloedema (18). 


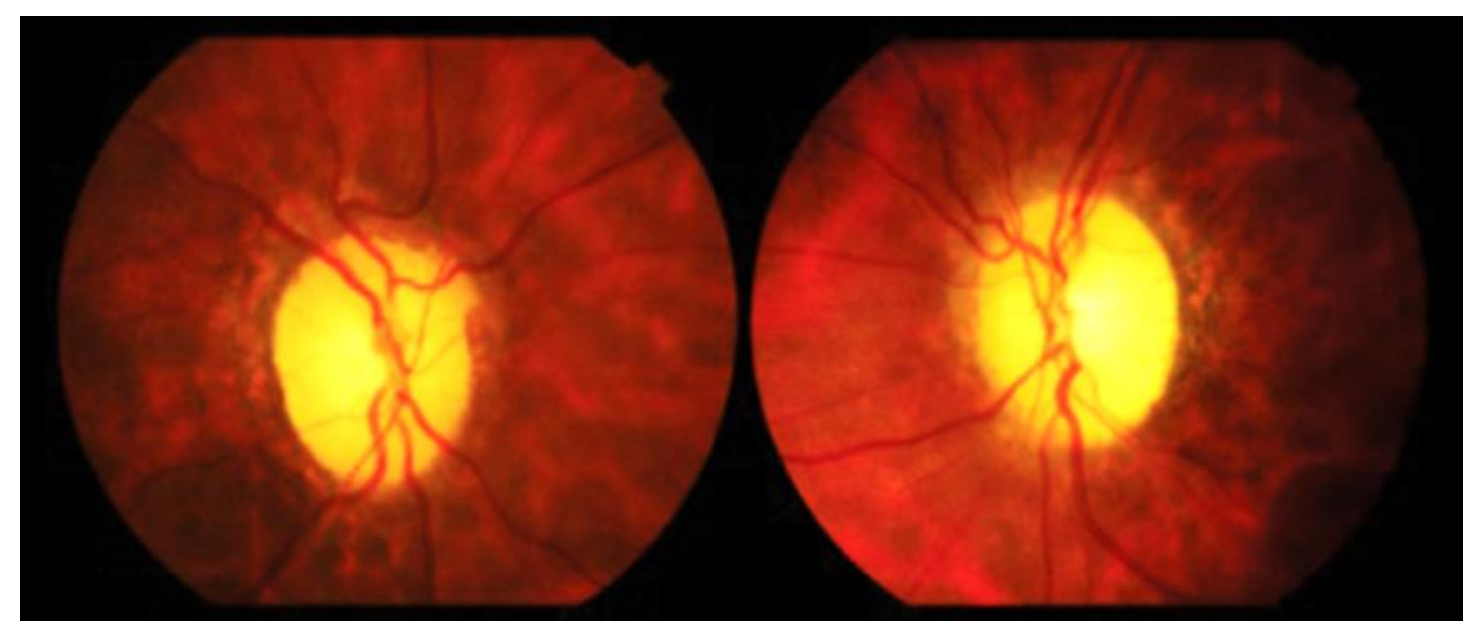

Figure (4): Secondary optic atrophy (19).

\section{Discussion}

Obesity has been associated with venous thromboembolism, perhaps mediated by release of procoagulant adipokines and other hormonal influences. This predisposition to hypercoagulability in obesity may lead to occult microthrombosis around the arachnoid granulations in the parasagittal venous lacunae, with subsequent impaired CSF outflow (7).

Two studies of IIH patients $(8,9)$ found a high prevalence of prothrombotic abnormalities, including antiphospholipid antibodies, abnormal protein $\mathrm{S}$ levels, Factor 5 Leiden, and prothrombin 20,210 mutations. One of the studies, however, showed that the prothrombotic conditions were more common in non-obese IIH patients (8) and the other study (9) controlled for age but not for obesity (a known risk factor for thromboembolism).

Occult thrombotic obstruction to CSF absorption also has been suggested to explain the link between IIH and polycystic ovarian syndrome (PCOS). In 25 patients with both IIH and PCOS done by Glueck and his co-authors in 2005 showed a high prevalence of thrombophilic abnormalities. 38\% had homozygous methylenetetrahydrofolate reductase mutations and $29 \%$ had thrombophilic increased lipoprotein A levels. Both abnormalities were 3 times more common in patients than in controls. Thrombophilic concentrations of factor VIII also occurred in $14 \%$ of the patients but in none of the controls (10).

Magnetic resonance imaging 3D volumetric study with dynamic phase- 
contrast imaging has demonstrated increased intracranial CSF volume within the extraventricular subarachnoid space along with reduced cerebral venous drainage through the internal jugular veins in obese IIH patients compared with obese controls. These findings suggest coexistence of impaired CSF homeostasis with altered venous hemodynamics in obesity-related IIH. Magnetic resonance imaging phase-contrast images of blood and CSF flow have also been used to noninvasively assess craniospinal compliance; significantly reduced spinal compliance was found in IIH subjects compared with obese matched controls. The authors postulate that an increased abdominal pressure from obesity limits the expansion of spinal canal CSF spaces, thereby reducing spinal compliance and altering CSF homeostasis. (11).

Another potential mechanism by which obesity may lead to raised ICP is alveolar hypoventilation or obstructive sleep apnea (OSA), both of which are associated with carbon dioxide retention, shown to increase ICP (12).

In a recent study (13), oligoclonal bands (OCBs) in serum and cerebrospinal fluid (CSF) were found in $31 \%$ of IIH patients. The authors discussed whether there might be some kind of association between obesity and OCB positivity, and whether obesity might lead to OCB positivity and IIH through an inflammatory process.

Recent study proposed a theory unifying various neuroendocrine effects on the mineralocorticoid receptor (MR) to explain a possible mechanism for the increased CSF production and ICP in secondary PTCS (14). The MR is abundant in choroid plexus (CP) epithelial cells, which regulate CSF production.

Increase in the activity in disorders of primary or secondary hyperaldosteronism may potentially directly affect the ICP in IIH. Support for this hypothesis includes numerous recent studies demonstrating that, in children and adults with primary or secondary hyperaldosteronism, those treated with spironolactone, an aldosterone receptor antagonist, had resolution of symptoms (15). Additional pediatric cases further strengthen this link of IIH associated with hyperaldosteronism whereby children have been successfully controlled with spironolactone after failure of acetazolamide treatment. In one case the child had secondary hyperaldosteronism, and in the other case the child had metabolic syndrome, obesity, and hyperaldosteronism (14). 
Regarding weight gain, Daniels et al. found that, in 34 newly diagnosed IIH patients and 41 controls, IIH patients reported higher degrees of weight gain in the 12 months prior to the onset of IIH. Higher BMI and percentages of weight gain were also associated with a dosedependent risk of developing IIH. Interestingly, both previously obese and non-obese patients (BMI <30) had a similar increased risk of developing IIH with only a small weight gain of 5-15\%.

The study suggests that the average weight of patients without recurrence was greater than that of patients with recurrence throughout the study, suggesting that weight change may be equally important in the development and resolution of IIH compared to baseline weight. Evidence supporting the importance of recent weight gain versus baseline weight also exists in the pediatric population (17).

Another study of 14 pediatric patients with IIH, all 5 patients with excess

The fact that weight loss improves or resolves signs and symptoms of IIH supports the strong association of obesity and IIH. While the etiology of IIH remains unknown, there have been many postulated theories. Proposed mechanisms that could explain the elevated ICP in IIH include increased cerebral volume (either interstitial fluid, blood or tissue), increased CSF volume due to increased production or resistance to CSF outflow, loss of cerebral autoregulation resulting in elevated cerebral arterial pressure or increased cerebral venous pressure leading to increased CSF volume and decreased CSF outflow.

There is still uncertainty as to whether the degree of obesity affects the severity of symptoms and disease outcomes, particular visual field defects. According to multiple studies, it appears that those with morbid obesity (BMI >40) have worse visual outcomes and this further underscores the importance of aggressively pursuing weight loss options in extremely obese patients.

As a whole, the data supports the role of weight loss in the treatment of IIH. Although patients may require other treatment modalities, given the benefits of even modest weight loss on IIH disease progression and outcomes, this should be an area of emphasis for treating physicians.

Weight loss can lead to remission of the symptoms of IIH. Management includes, but is not limited to, dietary and behavior modification incorporating routine physical activity. In fact, PTC/IIH in the 
setting of obesity may be curative with body weight loss of $10 \%$.

Although a number of treatments have been advocated, currently there is no evidence-based management strategy. Symptom relief is the primary goal of management, but achieving this goal and preventing morbidity may require a multifocal approach. Preserving visual function and headache relief- are priorities. Best practice would include an interdisciplinary team collaborating closely with a neurologist and ophthalmologist.

\section{Conclusion}

Weight loss can lead to remission of the symptoms of IIH. Management includes, but is not limited to, dietary and behavior modification incorporating routine physical activity. More studies are needed for more association between obesity \& IIH \& role of obesity management to reduce IIH.

\section{Sources of funding}

This research did not receive any specific grant from funding agencies in the public, commercial, or not-for-profit sectors.

\section{References}

1. Faz G, Butler IJ, Koenig MK. Incidence of papilledema and obesity in children diagnosed with idiopathic "“benign"'intracranial hypertension: case series and review. J Child Neurol. 2010;25(11):1389-92.

2. Molobe LT, Shifa J, Silava C, Mojela K, Cainelli F, Vento S. Primary pseudotumor cerebri syndrome in a young obese African woman. Isr Med Assoc J IMAJ. 2014;16(9):595-6.

3. Szewka AJ, Bruce BB, Newman NJ, Biousse V. Idiopathic intracranial hypertension: relation between obesity and visual outcomes. J neuro-ophthalmology Off J North Am Neuro-Ophthalmology Soc. 2013;33(1):4.

4. Kanski JJ, Bowling B. Clinical ophthalmology: a systematic approach. Elsevier Health Sciences; 2011.

5. Collins MLZ, Traboulsi EI, Maumenee IH. Optic Nerve Head Swelling and Optic Atrophy in the Systemic Mucopolysaccharides. Ophthalmology. 1990;97(11):1445-9.

6. Carter SR, Seiff SR. Macular changes in pseudotumor cerebri before and after optic nerve sheath fenestration. Ophthalmology. 1995;102(6):937-41.

7. Ooi L-Y, Walker BR, Bodkin PA, Whittle IR. Idiopathic intracranial hypertension: can studies of obesity provide the key to understanding pathogenesis? $\mathrm{Br} \mathrm{J}$ Neurosurg. 2008;22(2):187-94.

8. Dunkley S, Johnston I. Thrombophilia as a common predisposing factor in pseudotumor cerebri. Blood. 2004;103(5):1972-3.

9. Sussman J, Leach M, Greaves M, Malia R, Davies-Jones GA. Potentially prothrombotic abnormalities of coagulation in benign 
intracranial hypertension. J Neurol Neurosurg Psychiatry. 1997;62(3):229-33.

10. Subramaniam S, Fletcher WA. Obesity and weight loss in idiopathic intracranial hypertension: a narrative review. J Neuroophthalmology. 2017;37(2):197-205.

11. Alperin N, Ranganathan S, Bagci AM, Adams DJ, Ertl-Wagner B, Saraf-Lavi E, et al. MRI evidence of impaired CSF homeostasis in obesity-associated idiopathic intracranial hypertension. Am J Neuroradiol. 2013;34(1):29-34.

12. Banik R. Obesity and the role of nonsurgical and surgical weight reduction in idiopathic intracranial hypertension. Int Ophthalmol Clin. 2014;54(1):27-41.

13. Altıokka-Uzun G, Tüzün E, Ekizoğlu E, Ulusoy C, Yentür S, Kürtüncü $M$, et al. Oligoclonal bands and increased cytokine levels in idiopathic intracranial hypertension. Cephalalgia. 2015;35(13):1153-61.

14. Salpietro V, Polizzi A, Berte LF, Chimenz R, Chirico V, Manti S, et al. Idiopathic intracranial hypertension: a unifying neuroendocrine hypothesis through the adrenal-brain axis. Neuroendocr Lett. 2012;33(6):569-73.

15. Salpietro V, Chimenz R, Arrigo T, Ruggieri M. Pediatric idiopathic intracranial hypertension and extreme childhood obesity: a role for weight gain. $\mathrm{J}$ Pediatr. 2013;162(5).

16. Daniels AB, Liu GT, Volpe NJ, Galetta SL, Moster ML, Newman NJ, et al. Profiles of obesity, weight gain, and quality of life in idiopathic intracranial hypertension (pseudotumor cerebri). Am J Ophthalmol. 2007;143(4):635-41.

17. Ko MW, Chang SC, Ridha MA, Ney JJ, Ali TF, Friedman DI, et al. Weight gain and recurrence in idiopathic intracranial hypertension: a case-control study. Neurology. 2011;76(18):1564-7.

18. Hedges III TR, Legge RH, Peli E, Yardley CJ. Retinal nerve fiber layer changes and visual field loss in idiopathic intracranial hypertension. Ophthalmology. 1995;102(8):1242-7.

19. Cullen JF. Papilloedema: an update. Eye News. 2016;23(1):22-3.

To cite this article: Essam E. Saber, Ayser A. Fayed, Marwa A. Tabl, Ingy M. El Barki. Papilledema in Overweight Patients, BMFJ 2022;39 (ophthalmology):59-70. DOI: $10.21608 / \mathrm{bmfj} .2021 .20240 .1177$ 

Benha medical journal vol. 39, special issue (ophthalmology), 2022 